NEHU/PHY-MP-01/05

\title{
Threshold Effects on Quasi-degenerate Neutrinos with High-scale Mixing Unification
}

\author{
R. N. Mohapatre* \\ Department of Physics, University of Maryland, College Park, MD 20742, USA. \\ M. K. Paridat \\ Department of Physics, North-Eastern Hill University, Shillong 793022,India. \\ G. Rajasekaran \\ Institute of Mathematical Sciences, Chennai 600113, India.
}

(Dated: November 22, 2018)

\begin{abstract}
We consider threshold effects on neutrino masses and mixings in a recently proposed model for understanding large solar and atmospheric mixing angles using radiative magnification for the case of quasi-degenerate neutrinos. We show that the magnitude of the threshold effects is sufficient to bring concordance between the predictions of this model and latest data from KamLAND and SNO on observations of neutrino oscillations.

PACS numbers: 14.60.Pq, 11.30.Hv, 12.15.Lk
\end{abstract}

A major theoretical challenge posed by neutrino observations is how to understand the large difference between the mixing angles in lepton sector from that in the quark sector. In a recent paper [1] we proposed a mechanism based on the idea that neutrino masses and mixings derived in a seesaw framework are defined at high scale and, in order to compare them with experiments, we must extrapolate them to the weak scale using renormalization group equations [2]. It had been noted in ref. [3] that if neutrinos are quasi-degenerate and have same CP property, then small mixings at the seesaw scale can be "radiatively magnified" when extrapolated to the weak scale. The work of ref. 1] provided the first realistic model based on this idea. It started with the hypothesis that at the seesaw scale the quark and lepton mixing angles are identical and neutrinos are quasi-degenerate in mass with same CP property [1]. These assumptions were shown to be realizable in a class of very economical models based on $S U(4)_{c}$ gauge unification [4]. The individual neutrino masses (quasi-degenerate) are the inputs into the model and the rest are dictated by renormalization group equations of low energy MSSM. This led to large mixings in the solar and atmospheric neutrino sectors while maintaining consistency with the small upper limit on the mixing angle $\theta_{13}$ given by CHOOZ-Palo-Verde experiments. The precise values of the three input neutrino masses are determined by the present values of the mixing angles. The model has two interesting predictions: (i) it works only if the common mass of quasi-degenerate neutrinos is in the range $0.15 \mathrm{eV} \leq \mathrm{m}_{i} \leq 0.65 \mathrm{eV}$ and (ii) predicts that the lepton mixing angle $\sin \theta_{13} \leq 0.08-0.10$. The "large" value of the common mass is of great experimental interest since it falls in the range claimed by the Heidelberg-Moscow experiment [5] and is testable in very near future in all proposed $\beta \beta_{0 \nu}$ experiments [6]. It also has a significant overlap with the mass range accessible to the KATRIN experiment [7]. The predicted value for $\theta_{13}$ is also in the range accessible by experiments [8] being proposed. Therefore this mechanism for understanding large mixings is an eminently testable proposal.

While the predictions of the model are in good agreement with the gross features of the experimental data on atmospheric neutrinos and solar mixing angle, that for $\Delta m_{\odot}^{2}$ is larger than the latest value derived from the joint analysis of the KamLAND and solar neutrino data, which appeared subsequent to our work. In view of the fact that searches for neutrinoless double beta decay and $\theta_{13}$ are at the forefront of neutrino experimental efforts in the near future and since they also represent two crucial tests of our model, it is important to address this discrepancy and search for any other effects within the same model that can address this problem. In this brief report we point out such an effect.

In renormalizable field theories, there is indeed another effect that is relevant in comparing theory with experiment i.e. the one loop corrections that involve only the low scale physics. These are known as threshold effects. In our case it involves the one loop corrections to the extrapolated effective theory at the weak scale. These effects for neutrino masses have been discussed in [9] and shown to have a simple form if one assumes that the scalar masses are universal, as is usually done in MSSM to avoid conflict with flavor changing neutral current constraints. They have been found to make significant contribution on degenerate/quasi-degenerate neutrinos [9, 10]. In this note we estimate them in the high-scale mixing unification scenario.

Typeset by REVTEX 
In a typical seesaw model, the neutrino mass matrix at the weak scale can be written in the flavor basis as:

$$
\mathcal{M}_{\nu}(\mu)=I\left(M_{R}, \mu\right) \mathcal{M}_{\nu}^{0} I^{T}\left(M_{R}, \mu\right)+\Delta \mathcal{M}^{t h},
$$

where $\mathcal{M}_{\nu}^{0}$ is the neutrino mass matrix at the high (seesaw) scale and the renormalization effects from the seesaw scale $M_{\mathrm{R}}$ to the weak scale, $\mu$ are represented by a matrix in flavor space, $I\left(M_{R}, \mu\right)$ and the weak scale threshold effects are denoted by $\Delta \mathcal{M}^{\text {th }}$. In our model, the first term in Eq. (1) has already been calculated in ref. [1]. Using mass basis with $i, j=1,2,3$ denoting different mass eigenstates and representing the threshold corrections through loop factors by $T_{i j}$, the effects on mass eigenvalues in any model are expressed as $[9$, 10],

$$
m_{i j}=m_{i} \delta_{i j}+m_{i} T_{i j}+m_{j} T_{j i}
$$

It is clear that threshold effects are significant(negligible) for quasi-degenerate(hierarchical) neutrinos provided the masses are in the range of interest in our model (see above). As in [1], we ignore all phases for the sake of simplicity and parameterize the real $3 \times 3$ PMNS mixing matrix as

$$
U=\left[\begin{array}{ccc}
c_{13} c_{12} & c_{13} s_{12} & s_{13} \\
-c_{23} s_{12}-c_{12} s_{13} s_{23} & c_{12} c_{23}-s_{12} s_{13} s_{23} & c_{13} s_{23} \\
s_{12} s_{23}-c_{12} s_{13} c_{23} & -c_{12} s_{23}-c_{23} s_{13} s_{12} & c_{13} c_{23}
\end{array}\right],
$$

The loop factors in the mass basis can be expressed in terms of those in the flavor basis, $T_{\alpha \beta}(\alpha, \beta=e, \mu, \tau)$ leading to (for quasi-degenerate neutrinos)

$$
m_{i j}=m_{i} \delta_{i j}+2 m \sum_{\alpha, \beta} T_{\alpha \beta} U_{\alpha \mathrm{i}} U_{\beta \mathrm{j}} .
$$

where $\mathrm{m}$ is the common mass of quasi-degenerate neutrinos. In the MSSM flavor violation through threshold corrections is constrained to its minimal value through the diagonal structure of loop factors, $T_{\alpha \beta}=T_{\alpha} \delta_{\alpha \beta}$. Experimental data show that atmospheric neutrino mixing angle is close to its maximal value, $\theta_{\text {atm }}=\theta_{23} \simeq \pi / 4$ and the reactor mixing angle $\theta_{\mathrm{CHOOZ}}=\theta_{13}<10^{\circ}$ whereas the solar neutrino mixing angle deviates from its maximal value, $\theta_{\text {sol }}=\theta_{12} \simeq 31^{\circ}-34.5^{\circ}$. Then any result derived under the limiting case $\theta_{\text {atm }}=\pi / 4$ and $\theta_{\mathrm{CHOOZ}}=0$ is expected to hold in the actual case with a very good approximation. Using eqs.(1)-(3) and $\theta_{23}=\pi / 4, \theta_{13} \rightarrow 0$ the following relations are derived which are valid in any model under the assumption of minimal flavor violation:

$$
\begin{aligned}
\left(\Delta m_{21}^{2}\right)_{t h} & =4 \mathrm{~m}^{2} \cos 2 \theta_{12}\left[-\mathrm{T}_{\mathrm{e}}+\left(\mathrm{T}_{\mu}+\mathrm{T}_{\tau}\right) / 2\right], \\
\left(\Delta m_{32}^{2}\right)_{t h} & =4 \mathrm{~m}^{2} \sin ^{2} \theta_{12}\left[-\mathrm{T}_{\mathrm{e}}+\left(\mathrm{T}_{\mu}+\mathrm{T}_{\tau}\right) / 2\right], \\
\left(\Delta m_{31}^{2}\right)_{t h} & =4 \mathrm{~m}^{2} \cos ^{2} \theta_{12}\left[-\mathrm{T}_{\mathrm{e}}+\left(\mathrm{T}_{\mu}+\mathrm{T}_{\tau}\right) / 2\right],
\end{aligned}
$$

These equations in turn establish interrelations among threshold corrections to the three mass squared differences:

$$
\begin{array}{r}
\left(\Delta m_{21}^{2}\right)_{t h}=\left(\cot ^{2} \theta_{12}-1\right)\left(\Delta m_{32}^{2}\right)_{t h}, \\
=\left(1-\tan ^{2} \theta_{12}\right)\left(\Delta m_{31}^{2}\right)_{t h}, \\
\left(\Delta m_{32}^{2}\right)_{t h}=\tan ^{2} \theta_{12}\left(\Delta m_{31}^{2}\right)_{t h}, \\
\left(\Delta m_{21}^{2}\right)_{t h}-\left(\Delta m_{31}^{2}\right)_{t h}+\left(\Delta m_{32}^{2}\right)_{t h}=0,
\end{array}
$$

Eq. (11) says that the tree-level relation among the mass-squared differences is also true at one-loop level as is evident from Eqs. (8)-(10).

It is also clear from Eq. (8) that for allowed values of $\theta_{\odot}=32.6^{o} \pm 1.6^{o},\left(\Delta m_{21}^{2}\right)_{\mathrm{th}}=(5.5-1.2)\left(\Delta m_{32}^{2}\right)_{\mathrm{th}}$. Therefore any attempt to match the experimentally observed values of $\Delta m_{\text {atm }}^{2} \simeq 2 \times 10^{-3} \mathrm{eV}^{2}$ through $\left(\Delta m_{32}^{2}\right)_{t h}$ and $\Delta m_{\odot}^{2}$ through $\left(\Delta m_{21}^{2}\right)_{\text {th }}$ would lead to the prediction in the solar neutrino sector $\Delta m_{\odot}^{2} \approx 11.0 \times 10^{-3} \mathrm{eV}^{2}-2.4 \times 10^{-3} \mathrm{eV}^{2}$. Since these are at least two orders larger than the experimentally permissible range, we conclude that the low-energy threshold effects alone in any model can not accommodate the observed mass squared differences for solar and the atmospheric neutrinos.

Coming to the case of quasi-degenerate neutrino model of ref.[1], first point to note is that, there is more to the mass difference squares in this model than the threshold corrections. Therefore even though the $\left(\Delta m^{2}\right)_{t h}$ 's satisfy Eq. (5-7) they do not a priori lead to any contradiction with observations. For this case, it was shown in ref. [1] that for a wide range of GUT-seesaw scales, $M_{\mathrm{R}}=10^{11} \mathrm{GeV}-10^{18} \mathrm{GeV}$, one has the following predictions of the neutrino oscillation parameters: 


$$
\begin{aligned}
\left(\sin \theta_{12}\right)_{\mathrm{RG}} & =0.42-0.62,\left(\sin \theta_{23}\right)_{\mathrm{RG}}=0.660-0.707, \\
\left(\sin \theta_{13}\right)_{\mathrm{RG}} & =0.08-0.10,\left(\Delta m_{32}^{2}\right)_{\mathrm{RG}}=(1.18-4.6) \times 10^{-3} \mathrm{eV}^{2}, \\
\left(\Delta m_{21}^{2}\right)_{\mathrm{RG}} & =(1.20-6.0) \times 10^{-4} \mathrm{eV}^{2} .
\end{aligned}
$$

From the global fits of the solar neutrino data within $1 \sigma$ limit $\Delta m_{\odot}^{2}=(5-8) \times 10^{-5} \mathrm{eV}^{2}$. Thus, the predicted values of this quantity in the high-scale mixing unification model are at least $3 \sigma-4 \sigma$ larger while all other physical parameters are in good agreement with the experimental values. We show that quite reasonable and plausible threshold corrections due to super-partners at the electro-weak scale with minimal flavor violation which were ignored earlier are sufficient to account for this discrepancy. Other physical parameters undergo quite small or negligible threshold corrections while maintaining their agreement with the experimental data. Including threshold corrections along with the RG-evolution effects we define,

$$
\Delta m_{i j}^{2}=\left(\Delta m_{i j}^{2}\right)_{\mathrm{RG}}+\left(\Delta m_{i j}^{2}\right)_{\mathrm{th}}
$$

where, as stated through (12), the RG-evolution effects from $M_{\mathrm{R}}$ to $M_{\mathrm{Z}}$ have been already computed in [1]. We find that the simple form of the one-loop SUSY threshold effect with wino/slepton exchange in the loop is sufficient to give the desired correction. These effects due to gaugino/slepton exchange are evaluated using [10] and we find

$$
T_{\alpha}=\left(g^{2} / 32 \pi^{2}\right)\left[\left(x_{\mu}^{2}-x_{\alpha}^{2}\right) /\left(y_{\mu} y_{\alpha}\right)+\left(\left(y_{\alpha}^{2}-1\right) / y_{\alpha}^{2}\right) \ln \left(x_{\alpha}^{2}\right)-\left(\left(y_{\mu}^{2}-1\right) / y_{\mu}^{2}\right) \ln \left(x_{\mu}^{2}\right)\right]
$$

where $y_{\alpha}=1-x_{\alpha}^{2}, x_{\alpha}=M_{\alpha} / M_{\tilde{w}}, M_{\alpha}=$ charged slepton mass, and $M_{\tilde{w}}=$ wino mass. The loop-factor has been defined to give $T_{\mu}=0$ without any loss of generality [10]. For several allowed mass ratios $M_{\tilde{e}} / M_{\tilde{\mu}, \tilde{\tau}}$ in the MSSM we estimate threshold corrections $\left(\Delta m_{32}^{2}\right)_{t h}$ and $\left(\Delta m_{21}^{2}\right)_{t h}$ as shown in Table 1. We find that for inverted hierarchy in the charged-slepton sector with $M_{\tilde{\mathrm{e}}} / M_{\tilde{\mu}, \tilde{\tau}}=1.3-2.5$ the corrections are sufficient for agreement with the solar neutrino data within $1 \sigma$ limit. The correction to $\left(\Delta m_{32}^{2}\right)_{t h}$ is also negative but about one-order smaller than the corresponding RG contributions. The threshold corrections have negligible effects on the mixing angles obtained by RG-evolution. We give our detailed results in Table I.

We also observe that the inverted hierarchical form of neutrino mass eigenvalues, namely, $m_{2} \gtrsim m_{1} \gtrsim m_{3}$ is not allowed in the radiative magnification scenario[1]. We have found that in the MSSM with low-energy SUSY, the $\beta$-function coefficients of the three mass eigen values are positive near the see-saw scales with the third $\beta$-function coefficient being significantly larger compared to the first two. This is the basic reason why all the mass eigen values decrease although with different rates finally approaching a common value at the lowest-SUSY scale where magnification takes place. When applied to inverted hierarchical pattern, the mass eigenvalues move away from one another instead of approaching a common value at the SUSY scale.

In summary, we have calculated the threshold corrections to the neutrino masses and mixings in a high scale mixing unification model proposed recently to understand large mixings. We find that this substantially improves agreement of the model with the latest observations for $\Delta m_{\odot}^{2}$ as well as other oscillation observables. We have also argued on general grounds that both $\Delta m_{\odot}^{2}$ and $\Delta m_{a t m}^{2}$ can not be obtained by low-energy threshold effects alone on quasi-degenerate neutrinos.

This work was initiated at the Eighth Workshop on High-Energy Physics Phenomenology(WHEPP8), Mumbai, 2004. M.K.P. thanks the Institute of Mathematical Sciences, Chennai for Senior Associateship. The work of R.N.M is supported by the NSF grant No. PHY-0354401. The work of G.R. is supported by the DAE-BRNS Senior Scientist Scheme of the Govt. of India.

* Electronic address: rmohapat@physics.umd.edu

$\dagger$ Electronic address: mparida@sancharnet.in

‡ Electronic address: graj@imsc.res.in

[1] R. N. Mohapatra, M. K. Parida and G. Rajasekaran, hep-ph/ 0301234; Phys. Rev. D69, 053007 (20004).

[2] K.S. Babu, C.N. Leung and J. Pantaleone, Phys. Lett. B319, 191 (1993); P. Chankowski and Z. Pluciennik, Phys. Lett. B316, 312 (1993); S. Antusch, M. Drees, J. Kersten, M. Lindner and M. Ratz, Phys. Lett. B519, 238 (2001); Phys. Lett. B525, 130 (2002).

[3] K.R.S. Balaji, A.S. Dighe, R.N. Mohapatra and M.K. Parida, Phys. Rev. Lett. 84, 5034 (2000); Phys. Lett. B481, 33 $(2000)$.

[4] J. C. Pati and A. Salam, Phys. Rev. D10, 275 (1974). 
TABLE I: Radiative magnification to bilarge mixings and threshold correction effects at low energies for input values of $\mathrm{m}_{\mathrm{i}}^{0}(i=1,2,3), \sin \theta_{23}^{0}=0.038, \sin \theta_{13}^{0}=0.0025$, and $\sin \theta_{12}^{0}=0.22$ at the high scale $M_{R}$.

\begin{tabular}{|c|c|c|c|c|c|}
\hline$M_{\mathrm{R}}(\mathrm{GeV})$ & $10^{11}$ & $10^{13}$ & $10^{13}$ & $10^{15}$ & $2 \times 10^{18}$ \\
\hline$m_{1}^{0}(\mathrm{eV})$ & 0.4083 & 0.5170 & 0.6168 & 0.3980 & 0.5160 \\
\hline$m_{2}^{0}(\mathrm{eV})$ & 0.4100 & 0.5200 & 0.6200 & 0.4000 & 0.5200 \\
\hline$m_{3}^{0}(\mathrm{eV})$ & 0.4510 & 0.5910 & 0.7050 & 0.4730 & 0.6680 \\
\hline$m_{1}(\mathrm{eV})$ & 0.2723 & 0.3107 & 0.3719 & 0.2093 & 0.1714 \\
\hline$m_{2}(\mathrm{eV})$ & 0.2726 & 0.3122 & 0.3723 & 0.2098 & 0.1718 \\
\hline$m_{3}(\mathrm{eV})$ & 0.2745 & 0.3152 & 0.3759 & 0.2124 & 0.1750 \\
\hline$\left(\Delta m_{21}^{2}\right)_{\mathrm{RG}}\left(\mathrm{eV}^{2}\right)$ & $1.6 \times 10^{-4}$ & $3.0 \times 10^{-4}$ & $3.5 \times 10^{-4}$ & $2.0 \times 10^{-4}$ & $1.36 \times 10^{-4}$ \\
\hline$\left(\Delta m_{32}^{2}\right)_{\mathrm{RG}}\left(\mathrm{eV}^{2}\right)$ & $1.18 \times 10^{-3}$ & $1.8 \times 10^{-3}$ & $2.6 \times 10^{-3}$ & $1.15 \times 10^{-3}$ & $1.18 \times 10^{-3}$ \\
\hline$M_{\tilde{e}} / M_{\tilde{\mu}, \tilde{\tau}}$ & 1.5 & 1.9 & 2.3 & 1.9 & 1.5 \\
\hline$\left(\Delta m_{21}^{2}\right)_{\mathrm{th}}\left(\mathrm{eV}^{2}\right)$ & $-0.85 \times 10^{-4}$ & $-2.3 \times 10^{-4}$ & $-2.8 \times 10^{-4}$ & $-1.25 \times 10^{-4}$ & $-0.57 \times 10^{-4}$ \\
\hline$\left(\Delta m_{32}^{2}\right)_{\mathrm{th}}\left(\mathrm{eV}^{2}\right)$ & $-0.60 \times 10^{-4}$ & $-1.3 \times 10^{-4}$ & $-3.6 \times 10^{-4}$ & $-0.48 \times 10^{-4}$ & $-0.15 \times 10^{-4}$ \\
\hline$\Delta m_{\odot}^{2}\left(\mathrm{eV}^{2}\right)$ & $7.5 \times 10^{-5}$ & $7.0 \times 10^{-5}$ & $7.0 \times 10^{-5}$ & $7.0 \times 10^{-5}$ & $7.8 \times 10^{-5}$ \\
\hline$\Delta m_{\mathrm{atm}}^{2}\left(\mathrm{eV}^{2}\right)$ & $1.12 \times 10^{-3}$ & $1.7 \times 10^{-3}$ & $2.2 \times 10^{-3}$ & $1.1 \times 10^{-3}$ & $1.17 \times 10^{-3}$ \\
\hline $\sin \theta_{12}$ & 0.550 & 0.520 & 0.550 & 0.533 & 0.522 \\
\hline $\sin \theta_{23}$ & 0.700 & 0.707 & 0.690 & 0.695 & 0.696 \\
\hline $\sin \theta_{13}$ & 0.100 & 0.104 & 0.097 & 0.100 & 0.098 \\
\hline
\end{tabular}

[5] H.V. Klapdor-Kleingrothaus et al. Eur. Phys. J. A 12, 147 (2001); H. V. Klapdor-Kleingrothaus et al., Mod. Phys. Lett. A 16, 2409 (2001); hep-ph/0303217 H.V. Klapdor-Kleingrothaus, A. Dietz and I.V. Krivosheina, Foundations of Physics 32, 1181 (2002).

[6] C. Aalseth et al., arXiv:hep-ph/0412300

[7] A. Osipowicz et al.,(KATRIN Project), hep-ex/0109033

[8] K. Anderson et al., arXiv:hep-ex/0402041 M. Apollonio et al., Eur. Phys. J. C 27, 331 (2003) arXiv:hep-ex/0301017 M. V. Diwan et al., Phys. Rev. D 68, 012002 (2003) arXiv:hep-ph/0303081 D. Ayrea et al. hep-ex/0210005 Y. Itow et al. (T2K collaboration) hep-ex/0106019 I. Ambats et al. (NOVA Collaboration), FERMILAB-PROPOSAL-0929; M. Goodman, hep-ph/0501206

[9] E. J. Chun and S. Pokorski, Phys. Rev. D62,053001 (2000); P.Chankowski and P. Wasowicz, Eur. Phys. J. C23, 249 (2002); B. Brahmachari and E. J. Chun, hep-ph/0312030

[10] P. H. Chankowski, A. Ioannisian, S. Pokorski and J. W. F. Valle, Phys. Rev. Lett. 86, 3488 (2001). 